\section{D) Check for updates}

Cite this: Inorg. Chem. Front., 2020, 7, 4086

\title{
A metalloprotein-inspired thermo-gene for thermogels $\uparrow t$
}

\author{
Kaerdun Liu, Cheng Ma, Wenkai Wang, (D) Shihao Zang, Yiteng Cai, Wei Chen, \\ Zeyu Liu, Jianbin Huang and Yun Yan (D)*
}

Physical hydrogels and many chemical hydrogels usually melt at temperatures higher than $40-50{ }^{\circ} \mathrm{C}$. We show this by introducing a coordinating hydrogel as a thermo-gene. Heat-melting hydrogels can be transformed into thermogels that remain stable at temperatures over $80^{\circ} \mathrm{C}$. Devices made with this gel work stably at a high temperature of $80^{\circ} \mathrm{C}$. In combination with previous knowledge of anti-freezing hydrogels, the current work allows us to remove the bottleneck that limits the practical application of hydrogel-based flexible devices under different weather conditions.

Received 1st July 2020,

Accepted 10th August 2020

DOI: 10.1039/d0qi00788a

rsc.li/frontiers-inorganic

\section{Introduction}

Hydrogels composed of fixed water in the physically crosslinked networks of self-assembled fibers or polymer chains, namely physical hydrogels, have emerged as powerful materials in a wide range of fields, such as biology,${ }^{1-3}$ pharmacy $^{4,5}$ and materials science. ${ }^{6-8}$ Recent studies revealed that physical hydrogels are suitable materials for various flexible devices including wearable sensors, ${ }^{9-12}$ stretchable displays, ${ }^{13,14}$ and flexible supercapacitors ${ }^{15-17}$ owing to their good flexibility, stretchability and conductivity. Unfortunately, the devices made of physical hydrogels can work only in a very narrow temperature range because most hydrogels freeze as the temperature falls below $0{ }^{\circ} \mathrm{C}$ and melt into viscous solution at above the gel-to-sol temperature $\left(T_{\text {gel }}\right)$ around $40-50{ }^{\circ} \mathrm{C} .{ }^{18}$ These conditions cause fragility or collapse of the physical networks, making them lose their original flexibility and mechanical strength. Therefore, there is a very urgent need to improve both the cold and heat resistance of physical hydrogels for practical applications.

Recently, scientists have developed a number of anti-freezing hydrogels using binary solvent systems (ethylene glycolwater or glycerol-water ${ }^{19,20}$ ) or inorganic salts, ${ }^{8,21}$ which are inspired from the anti-freezing mechanism of peeper frogs and rainbow fish in extremely cold weather. However, stable physical hydrogels that can tolerate high temperatures, which

Beijing National Laboratory for Molecular Sciences (BNLMS), College of Chemistry and Molecular Engineering, Peking University, Beijing, 100871, P. R. China. E-mail:yunyan@pku.edu.cn

$\dagger$ Electronic supplementary information (ESI) available: Materials, fabrication and additional figures. See DOI: 10.1039/d0qi00788a

$\$$ In celebration of the 110th anniversary of the College of Chemistry and Molecular Engineering of Peking University. we named thermogels (TG), still remain insurmountable. Actually, in nature, there are organisms capable of tolerating extremely hot conditions by production of desired substances in their body. For example, thermophile and heat-loving microbes employ metalloproteins to resist high temperature. ${ }^{22-24}$ When most proteins denature at temperatures beyond $40-50^{\circ} \mathrm{C}$, the structure of metalloproteins remains stable. A recent study has shown that coordination-mediated cutinase can even catalyze the hydrolysis of plastics at a high temperature of $72{ }^{\circ} \mathrm{C}^{25}$ This provides inspiration that the coordination interaction may also be employed in physical hydrogels and other heat-melting gels in a planned way to endow the gels with high temperature resistance. Some metallo-hydrogels in the literature indeed show excellent thermo-resistance. ${ }^{26-28}$ However, so far the role of metal coordination in the thermal stability of hydrogels has not been systematically studied.

Herein, we report a metalloprotein-inspired design of a TG that would endow heat-melting hydrogels with thermal stability. We found that upon introducing a metallo-hydrogel into any conventional hydrogels, the resultant hydrogel would inherit the thermo-resistance of the metallo-hydrogels. In this sense, the metallo-hydrogel behaves like a 'gene' that expresses itself in other gels, and we named it 'thermo-gene'. The thermo-gene is the coordinating hydrogel formed with folate and zinc ions (Scheme 1a). Previous work verified that the coordination between the carbonate groups of the folate and $\mathrm{Zn}^{2+}$ ions in water would lead to printable hydrogels, ${ }^{29}$ where the folate ions form tetramers, which further stack into fibers via $\pi-\pi$ interactions. These fibers were then cross-linked by the coordination between $\mathrm{Zn}^{2+}$ and the carboxylate groups on the fiber surface to form bundles and physical networks ${ }^{29}$ (Scheme 1a). In this work, we show that the thermo-gene itself is a TG, and this TG remains stable even at a high temperature of $90{ }^{\circ} \mathrm{C}$. Strikingly, it is capable of transforming various heat-melting hydrogels into TGs when intro- 

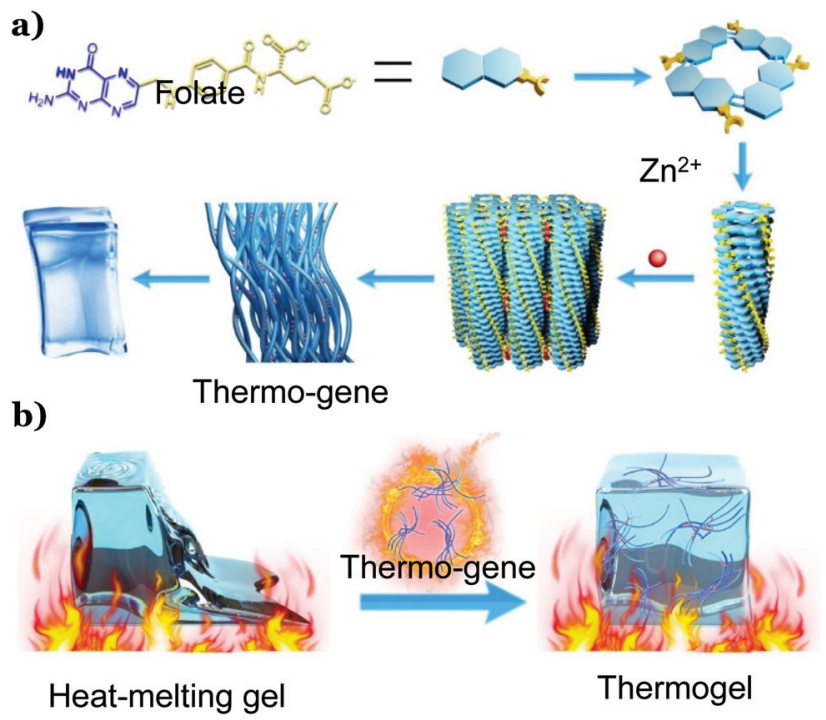

Scheme 1 Illustration of the folate- $\mathrm{Zn}^{2+}$ hydrogel thermo-gene (a) and the formation of a thermogel by introducing the thermo-gene into a heat-melting gel (b).

duced as the second network (Scheme 1b). All the resultant double network thermogels (DNTGs) inherit the excellent thermal stability of the thermo-gene. The stress sensor made with this DNTG works stably even at a high temperature of $80{ }^{\circ} \mathrm{C}$ when most physical hydrogels and many chemical hydrogels melt into solutions. ${ }^{30}$ In combination with the antifreezing technique developed in previous studies, ${ }^{8,19-21}$ the working temperature window of the DNTG can be extended from sub-zero degrees to around $90{ }^{\circ} \mathrm{C}$. As such, we expect that this metalloprotein-inspired thermo-gene would help solve the poor heat resistance problem in the development of hydrogelbased flexible devices, paving the way for their practical application in both extremely cold and extremely hot weather.

\section{Results and discussion}

Figure 1a shows the temperature scanning rheology measurement for the thermo-gene of the folate- $\mathrm{Zn}^{2+}$ hydrogel. Its characteristic gelling behavior was observed in a wide temperature range of $30-80{ }^{\circ} \mathrm{C}$. Higher temperature is not adopted to avoid drying of the gels in the measurement process. At all the tested temperatures, the shear modulus did not drop. Instead, a slight increase of the shear modulus was observed in the temperature range of $30-50{ }^{\circ} \mathrm{C}$, indicating that the physical network is evenly strengthened. ${ }^{31}$ This strengthened state is maintained at higher temperatures, which is evidenced by the constant shear modulus at $50-80{ }^{\circ} \mathrm{C}$. In fact, the gel still remains solid-like at $90{ }^{\circ} \mathrm{C}$ (inset in Fig. 1a). Detailed rheological measurements suggested that the storage modulus (elastic modulus, $G^{\prime}$ ) is 1 order higher than the loss modulus (viscous modulus, $G^{\prime \prime}$ ) at all the tested temperatures (Fig. S1†), confirming that the network in the hydrogel remains stable. In line
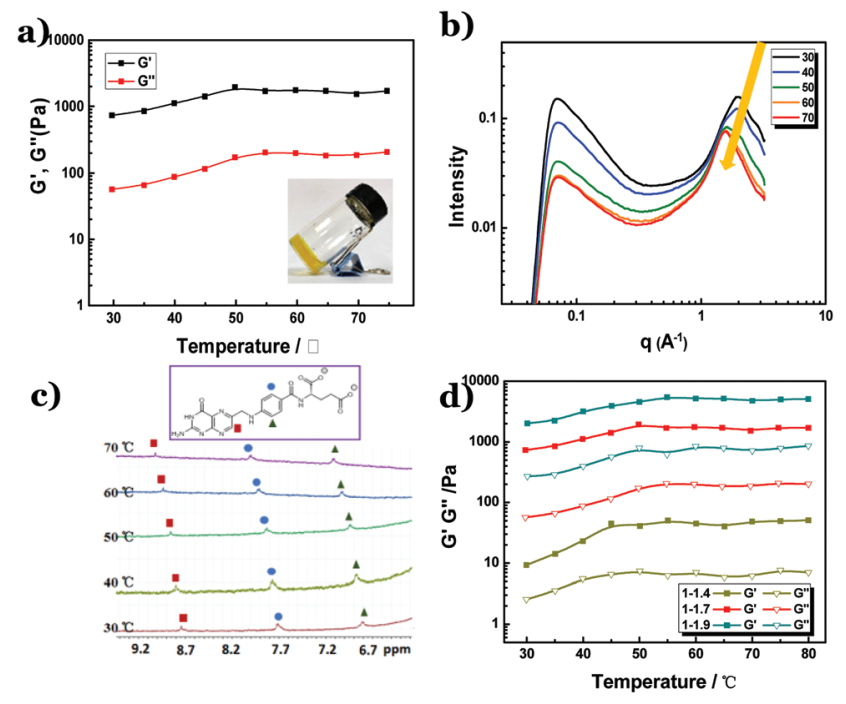

Fig. 1 (a) Rheological measurements of the folate $-\mathrm{Zn}^{2+}$ hydrogel in the temperature range of $30{ }^{\circ} \mathrm{C}-80^{\circ} \mathrm{C}$. Higher temperature is not adopted to avoid the drying of the gels in the measurement process. The inset is a photograph of the gel at $90^{\circ} \mathrm{C}$. (b) SAXS spectra of the folate- $\mathrm{Zn}^{2+}$ hydrogel with rising temperature. The numbers in the legend are temperatures in ${ }^{\circ} \mathrm{C}$. The arrow is to guide the direction of the peak shifting. (c) ${ }^{1} \mathrm{HNMR}$ spectra of the folate- $\mathrm{Zn}^{2+}$ hydrogel with rising temperature. Sample conditions in SAXS and ${ }^{1} \mathrm{H}$ NMR: [folate] $=15 \mathrm{mM}$, [folate] : $\left[\mathrm{Zn}^{2+}\right]=1: 1.7$. $($ (d) Temperature-dependent rheological measurements of folate- $\mathrm{Zn}^{2+}$ hydrogels at a given [folate] of $15 \mathrm{mM}$ but varied [folate] : $\left[\mathrm{Zn}^{2+}\right]$ molar ratios.

with the rheological results, the circular dichroism (CD) spectra of the hydrogel were not affected by the variation of temperature, too (Fig. S2 $\dagger$ ), indicating that the chiral stacking of petrin tetramers remains unchanged. However, SAXS measurements revealed that the distance between the folate tetramers increased from $3.18 \AA$ to $4.08 \AA$ with a temperature increase from $30{ }^{\circ} \mathrm{C}$ to $70{ }^{\circ} \mathrm{C}$, which is evidenced by the shift of the scattering peak from $q=1.97 \AA^{-1}$ to $1.54 \AA^{-1}$ (Fig. 1b). Meanwhile, the ${ }^{1}$ HNMR peaks are broadened at all the tested temperatures, indicative of the presence of effective $\pi-\pi$ stacking at both low and high temperatures. However, the chemical shifts of the protons on the petrin ring in the ${ }^{1} \mathrm{H}$-NMR spectra move toward a lower field (Fig. 1c), which means that the electron density on the petrin ring is reduced. ${ }^{32}$ In combination with the SAXS and HNMR measurements, it is obvious that the $\pi-\pi$ interaction is weakened by the increase in temperature. It is then clear that the coordination interaction has played a crucial role in reserving the physical network in the hydrogel, just like metalloproteins in thermophiles.

To further confirm the role of coordination in endowing the folate- $\mathrm{Zn}^{2+}$ hydrogel with thermal stability, a variation of the extent of coordination was conducted in the gel system. Fig. $1 \mathrm{~d}$ shows that as the molar ratio of folate: $\mathrm{Zn}^{2+}$ varies from $1: 1.4$ to $1: 1.9$, all the resulting hydrogels display thermal resistance. An increased fraction of $\mathrm{Zn}^{2+}$ leads to a higher shear modulus, verifying that the increased extent of coordination linkages would strengthen the networks in the 
hydrogel. It is noteworthy that the shear modulus in all the tested hydrogels increases in the temperature range of 30-50 ${ }^{\circ} \mathrm{C}$, and then levels off at higher temperatures. According to the analysis in the previous text, this temperature-triggered increase of shear modulus indicates that the coordination interaction is promoted upon increasing the temperature. Indeed, TEM observation reveals that the diameter of the nanofibers at $80{ }^{\circ} \mathrm{C}$ is larger than that at room temperature (Fig. S $3 \dagger$ ).

Since the coordination interaction between folate and $\mathrm{Zn}^{2+}$ is very strong, it would not be interfered with by non-coordinating components. We therefore expect that the folate- $\mathrm{Zn}^{2+}$ network would act as a 'thermo-gene' for heat-melting gels to render them superior thermal stability. To this end, the stock solutions of folate and $\mathrm{Zn}^{2+}$ were introduced to the melts of three types of heat-melting hydrogels covering both physically and chemically cross-linked ones. Two physically cross-linked hydrogels were employed, including (1) the freezing-thawing gel formed by polyvinyl alcohol (PVA) and (2) the heatingcooling gel of agarose. The last one (3) is the chemically crosslinked hydrogel of in situ polymerized polyacrylamide (PAM). To prepare this chemical hydrogel, the solutions of folate, $\mathrm{Zn}$ $\left(\mathrm{NO}_{3}\right)_{2}$, and an acrylamide (AM) monomer were premixed, followed by the addition of initiators ammonium persulfate (APS) and tetramethylethylenediamine (TEMED). Doublenetwork $^{33,34}$ thermogels (DNTGs) were formed in all the above systems (Fig. 2a-c), and they all displayed solid-like characteristics at temperatures up to $90{ }^{\circ} \mathrm{C}$ (Fig. 2d-f). In contrast, all the corresponding single polymer gels melted at temperatures around $60^{\circ} \mathrm{C}$ (Fig. 2g-i). DNTGs would be formed by replacing $10 \%$ of the heat-melting gel with the thermo-gene folate- $\mathrm{Zn}^{2+}$ gel, and increasing the concentration of the thermo-gene (Fig. $\mathrm{S} 4 \dagger$ ) or the fraction of $\mathrm{Zn}^{2+}$ (Fig. 2j) would increase the shear modulus of the DNTG considerably. These results unambiguously confirm that the coordination interaction is critical for the temperature resistance.

In order to understand how the thermo-gene folate- $\mathrm{Zn}^{2+}$ hydrogel enhanced the temperature resistance of the DNTG, the microstructure of the DNTG is compared with that of single hydrogels. Fig. 3 shows the SEM images of the three freeze-dried gels of folate- $\mathrm{Zn}^{2+}$, PVA, and the DNTG of folate$\mathrm{Zn}^{2+} /$ PVA. The dried thermo-gene folate- $\mathrm{Zn}^{2+}$ is a continuous structure with large pores (Fig. 3a), where the walls of the pores are composed of bundles of fibers (Fig. S5†). Different from these porous structures, the dried PVA gel is composed of individual fibers (Fig. 3b). It is interesting to find that the DNTGs perfectly inherit the characteristics of each single component in a manner of hosting fibers in large pores (Fig. 3c). Elemental mapping of the DNTG reveals that there are holes of $\mathrm{Zn}^{2+}$ distribution, as indicated by the red circles in Fig. 3d. This means that the thermo-gene folate- $\mathrm{Zn}^{2+}$ has formed an entire network in the DNTG, which acts as a strong skeleton to maintain the DNTG as the temperature increases. Actually, the PVA network tends to melt in the DNTG upon heating, which can be inferred from the sharpening and down-field shifting of the ${ }^{1}$ HNMR signals for the PVA protons (Fig. 3e). However,
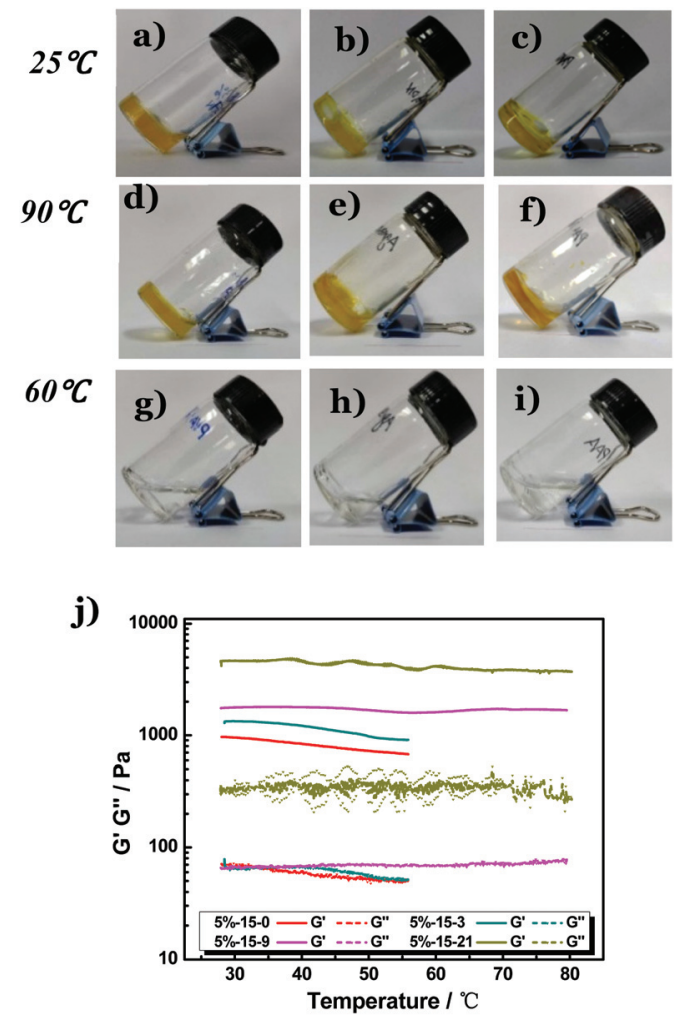

Fig. 2 Impact of the thermo-gene folate- $\mathrm{Zn}^{2+}$ on different heatmelting hydrogels. (a-c) The DNTG of PVA/folate- $\mathrm{Zn}^{2+}(w t \% 9: 1, a)$, agarose/folate- $\mathrm{Zn}^{2+}$ (wt\% $\left.9: 1, \mathrm{~b}\right)$, and PAM/folate- $Z \mathrm{n}^{2+}(w t \% 9: 1, \mathrm{c})$ at $25{ }^{\circ} \mathrm{C}$. (d-f) The corresponding DNTG at $90{ }^{\circ} \mathrm{C}$. ( $\left.\mathrm{g}-\mathrm{i}\right)$ The single gel of $10 \%$ PVA, agarose, and PAM at $60{ }^{\circ} \mathrm{C}$. (j) Rheological measurements of the DNTG with a varied molar ratio of [folate] : $\left[\mathrm{Zn}^{2+}\right] .[\mathrm{PVA}]=5 \%$, [folate] $=15 \mathrm{mM}$, and the concentrations of $\left[\mathrm{Zn}^{2+}\right]$ are $0,3,9$, and $21 \mathrm{mM}$.

the extent of the PVA network melting in the DNTG is reduced, since the chemical shifts in the DNTG system are much smaller than that in the single PVA gel (Fig. S6†). It is clear that the presence of folate- $\mathrm{Zn}^{2+}$ not only endows the system with a thermally resistant network but also enhances the stability of the PVA network. These two facts co-function as the DNTG is heated, which finally endows the DNTG with excellent temperature tolerance.

Along with the superior thermal resistance, the DNTG also displays enhanced mechanical strength. ${ }^{35,36}$ The folate- $\mathrm{Zn}^{2+}$ gel is not stretchable, while the PVA/folate- $\mathrm{Zn}^{2+}$ DNTG can be stretched to nearly $1000 \%$ with a stress of $1706 \mathrm{kPa}$ (Fig. 4a and b). In contrast, the original PVA gel can only be stretched to $750 \%$ with a stress of $1000 \mathrm{kPa}$. This high stretchability allows the PVA/folate- $\mathrm{Zn}^{2+}$ DNTG to resist blade cutting (Fig. 4c), which is a valuable merit desired for robust flexible devices. It is noteworthy that this excellent mechanical property of the PVA/folate- $\mathrm{Zn}^{2+}$ gel can extend to very high temperatures. Fig. 4d-f show that the shape, volume, and height of the $\mathrm{PVA} /$ folate- $\mathrm{Zn}^{2+}$ DNTG can recover after compression at $80^{\circ} \mathrm{C}$, manifesting its excellent application potential in thermally stable flexible devices. 

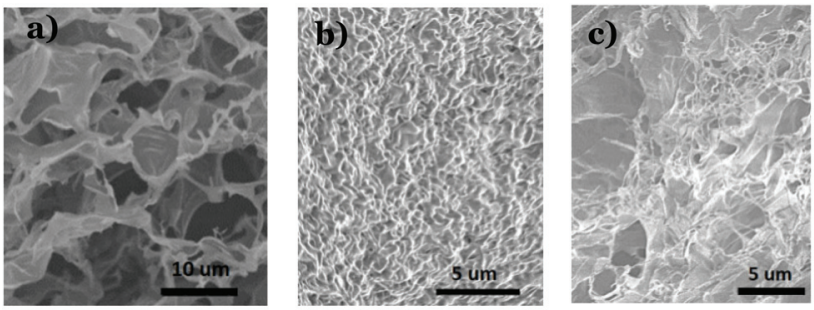

d)

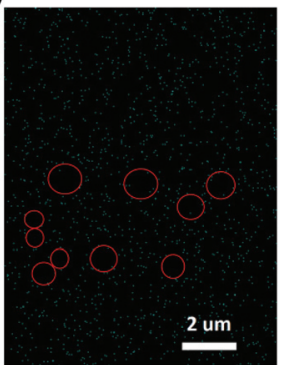

e)

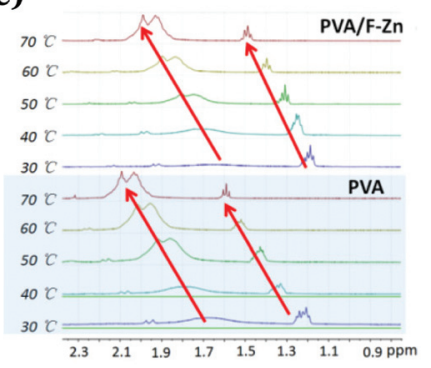

Fig. 3 Microstructure of the DNTG. (a-c) SEM image of the microstructure of the folate- $\mathrm{Zn}^{2+}$ gel, PVA gel, and the PVA/folate- $\mathrm{Zn}^{2+}$ DNTG, respectively. (d) The elemental mapping of zinc in the dried DNTG. The red circles identify the pores where no $\mathrm{Zn}^{2+}$ is present. (e) The ${ }^{1} \mathrm{HNMR}$ spectra for the protons of a single PVA gel and PVA/F- $Z n^{2+}$ DNTG. F-Zn represents the folate- $\mathrm{Zn}^{2+}$ hydrogel. The arrows are to guide the direction of the chemical shift of the $\mathrm{CH}_{2}$ proton in PVA.

a)
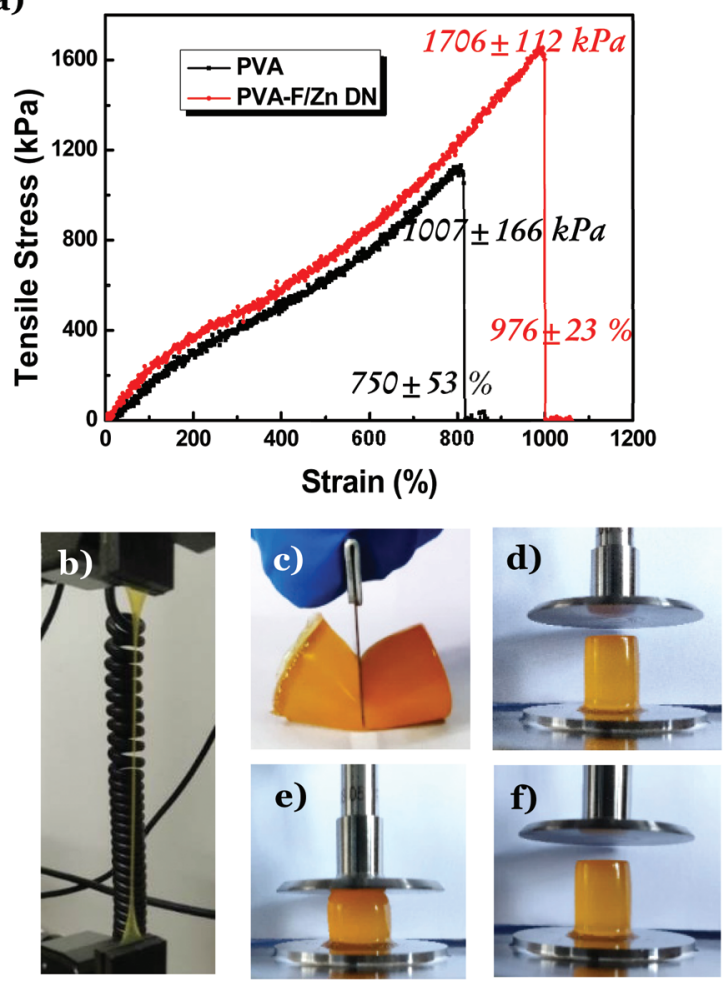

Fig. 4 Mechanical measurements of the PVA/folate $-\mathrm{Zn}^{2+}$ DNTG. (a) Tensile stress-elongation curve of a single PVA gel and the PVA/folate$\mathrm{Zn}^{2+}$ DNTG. (b) Photograph of the PVA/folate- $\mathrm{Zn}^{2+}$ DNTG during strain (around $800 \%$ ). (c) PVA/folate- $Z n^{2+}$ DNTG can endure the cutting. (d)(f) PVA/folate- $\mathrm{Zn}^{2+}$ DNTG in the compression-recovery cycle at $80^{\circ} \mathrm{C}$.
Next, a DNTG film was prepared to fabricate flexible sensors. Because of the presence of potassium and nitrate ions in the folate- $\mathrm{Zn}^{2+}$ hydrogel, the resultant DNTG displays excellent conductivity (Fig. S7†). The conductivity can be further promoted by preparing the DNTG in aqueous solutions of inorganic salts, such as $\mathrm{KNO}_{3}$ (Fig. S8 $\dagger$ ). By filling the PVA/folate- $\mathrm{Zn}^{2+}$ melt in a self-made thin layer mold, a $100 \mu \mathrm{m}$-thick gel film was obtained (Fig. 5a). Fig. 5b shows that the film displays excellent flexibility and elasticity, manifesting great potential in application as stress sensors. Indeed, as a stripe of the gel film (Fig. S9a, inset†) was introduced to a circuit (Fig. S9a†) and attached to a human finger (Fig. S9b $\dagger$ ), bending and stretching of the finger resulted in a significant current change and electronic resistance change (Fig. S9c $\dagger$ ). These changes are completely reversible with repeated finger bending and stretching movements, and the amplitude of the current change is proportional to the extent of the bending action, indicating the excellent sensitivity of the flexible stress sensor.

Most strikingly, this flexible stress sensor is stable even at high temperatures. For the convenience of measurements, we attached the film to a steel plate. To avoid short circuit, the steel plate was covered with an insulated plastic (Fig. 5c). Fig. 5d (left) shows that as the plate is heated to $80^{\circ} \mathrm{C}$, the film
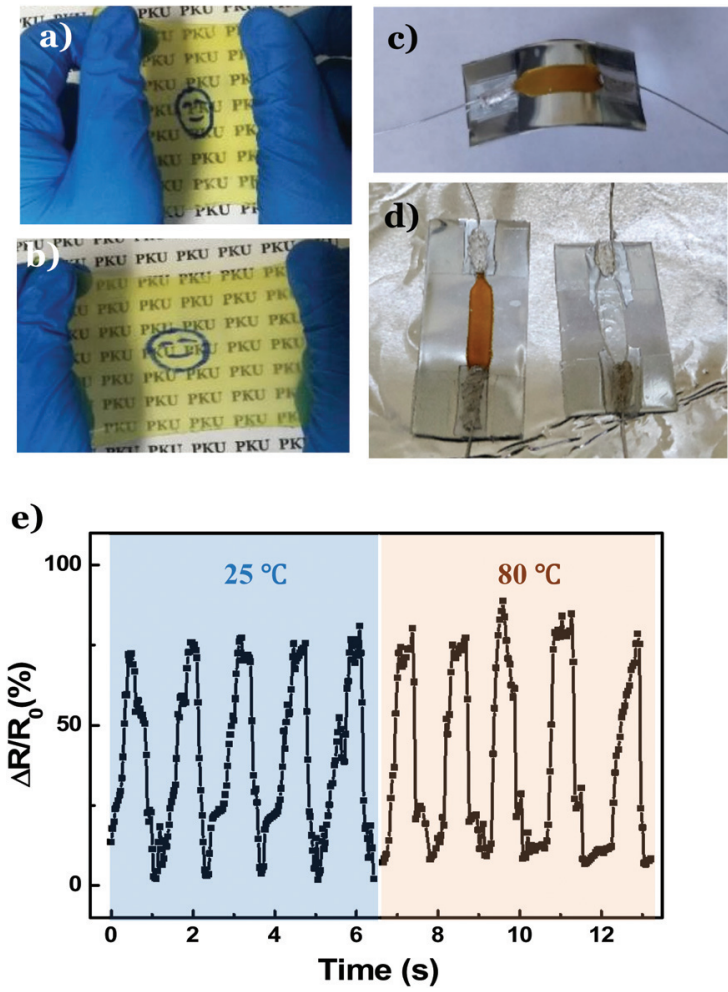

Fig. 5 Stress sensor made with PVA/folate- $\mathrm{Zn}^{2+}$ DNTG. (a) Photograph of the flexible DNTG film and (b) stretching the film. (c) Photographs of the DNTG sensor on a steel plate at room temperature $\left(25^{\circ} \mathrm{C}\right)$. (d) The DNTG sensor at $80^{\circ} \mathrm{C}$ (left) remains stable, whereas the PVA sensor at $60{ }^{\circ} \mathrm{C}$ (right) starts to melt. (e) Variation of the electrical resistance of the stress sensor during repeated bending-stretching deformations at $25^{\circ} \mathrm{C}$ and $80^{\circ} \mathrm{C}$. 
sensor remains intact, and a reversible current change is observed as the steel plate is repeatedly bent (Fig. 5e). In contrast, the stress sensor made from the PVA gel melted at $60{ }^{\circ} \mathrm{C}$ (Fig. 5d, right). This result unambiguously verifies that the DNTG can be used as a robust stress sensor that may work in an extremely hot environment.

It is noteworthy that the 'thermo-gene' does not interfere with the anti-freezing ability of the polymer hydrogels. If the solvent water is replaced with a mixture of glycol and a water binary system, the DNTG (Fig. S10†) also displays an anti-freezing ability. Fig. S11† shows that the storage modulus (elastic modulus, $G^{\prime}$ ) and the loss modulus (viscous modulus, $G^{\prime \prime}$ ) of the DNTG of PVA/ folate- $\mathrm{Zn}$ are nearly constant in a wide temperature range of $-30^{\circ} \mathrm{C}$ to $80^{\circ} \mathrm{C}$, whereas for the single PVA gel they decrease with elevated temperature. This indicates that the strategy of the DNTG can be jointly employed with the anti-freezing technique, allowing the fabrication of flexible hydrogel devices that can work from sub-zero degrees to temperatures exceeding $80^{\circ} \mathrm{C}$.

\section{Conclusions}

In conclusion, the coordinating folate- $\mathrm{Zn}^{2+}$ hydrogel can be used as a thermo-gene to endow heat-melting hydrogels with thermal stability via a double network strategy. The resultant double network thermogels (DNTGs) perfectly inherit the thermal stability of the folate- $\mathrm{Zn}^{2+}$ hydrogel, so that the flexible conductive sensor made with the DNTG works stably up to a high temperature of $80{ }^{\circ} \mathrm{C}$ where most physical hydrogels and some chemical hydrogels melt. In combination with the antifreezing technique, this work indicates that the working temperature window of hydrogels can be broadened from sub-zero degrees to temperatures exceeding $80{ }^{\circ} \mathrm{C}$. We envision that the coordinating hydrogels could be universal thermo-genes capable of endowing the heat-melting hydrogels with thermal stability, which is advantageous for the practical applications in smart devices. Relevant work is ongoing in our laboratory.

\section{Conflicts of interest}

There are no conflicts to declare.

\section{Acknowledgements}

The authors are grateful to the National Natural Science Foundation of China (Grant No. 91856120 and 21633002) and the Beijing National Laboratory for Molecular Sciences (BNLMS) for financial support.

\section{Notes and references}

1 J. L. Drury and D. J. Mooney, Hydrogels for tissue engineering: scaffold design variables and applications, Biomaterials, 2003, 24(24), 4337-4351.
2 H. Wang and S. C. Heilshorn, Adaptable hydrogel networks with reversible linkages for tissue engineering, Adv. Mater., 2015, $27(25)$, 3717-3736.

3 Z. J. Fan, B. Liu, J. Q. Wang, S. Y. Zhang, Q. Q. Lin, P. W. Gong, L. M. Ma and S. R. Yang, A novel wound dressing based on Ag/graphene polymer hydrogel: effectively kill bacteria and accelerate wound healing, Adv. Funct. Mater., 2014, 24(25), 3933-3943.

$4 \mathrm{~J}$. Li and D. J. Mooney, Designing hydrogels for controlled drug delivery, Nat. Rev. Mater., 2016, 1(12), 16071.

5 Y. Qiu and K. Park, Environment-sensitive hydrogels for drug delivery, Adv. Drug Delivery Rev., 2001, 53(3), 321-339.

6 J. Y. Sun, X. Zhao, W. R. Illeperuma, O. Chaudhuri, K. H. Oh, D. J. Mooney, J. J. Vlassak and Z. Suo, Highly stretchable and tough hydrogels, Nature, 2012, 489(7414), 133-136.

7 T. L. Sun, T. Kurokawa, S. Kuroda, A. B. Ihsan, T. Akasaki, K. Sato, M. A. Haque, T. Nakajima and J. P. Gong, Physical hydrogels composed of polyampholytes demonstrate high toughness and viscoelasticity, Nat. Mater., 2013, 12(10), 932-937.

8 X. Zhao, F. Chen, Y. Li, H. Lu, N. Zhang and M. Ma, Bioinspired ultra-stretchable and anti-freezing conductive hydrogel fibers with ordered and reversible polymer chain alignment, Nat. Commun., 2018, 9, 3579.

9 C. H. Yang and Z. G. Suo, Hydrogel ionotronics, Nat. Rev. Mater., 2018, 3(6), 125-142.

10 Y. Si, L. Wang, X. Wang, N. Tang, J. Yu and B. Ding, Ultrahigh-water-content, superelastic, and shape-memory nanofiber-assembled hydrogels exhibiting pressureresponsive conductivity, Adv. Mater., 2017, 29(24), 1700339.

11 G. Cai, J. Wang, K. Qian, J. Chen, S. Li and P. S. Lee, Extremely stretchable strain sensors based on conductive self-healing dynamic cross-links hydrogels for humanmotion detection, Adv. Sci., 2017, 4(2), 1600190.

12 X. Wang, Z. Liu and T. Zhang, Flexible sensing electronics for wearable/attachable health monitoring, Small, 2017, 13(25), 1602790.

13 C. C. Kim, H. H. Lee, K. H. Oh and J. Y. Sun, Highly stretchable, transparent ionic touch panel, Science, 2016, 353(6300), 682-687.

14 L. Shi, T. Zhu, G. Gao, X. Zhang, W. Wei, W. Liu and S. Ding, Highly stretchable and transparent ionic conducting elastomers, Nat. Commun., 2018, 9(1), 2630.

15 S. Ghosh and O. Inganas, Conducting polymer hydrogels as 3D electrodes: Applications for supercapacitors, Adv. Mater., 1999, 11(14), 1214-1218.

16 Y. Xu, Z. Lin, X. Huang, Y. Wang, Y. Huang and X. Duan, Functionalized graphene hydrogel-based high-performance supercapacitors, Adv. Mater., 2013, 25(40), 5779-5784.

17 Y. Huang, M. Zhong, F. Shi, X. Liu, Z. Tang, Y. Wang, Y. Huang, H. Hou, X. Xie and C. Zhi, An Intrinsically stretchable and compressible supercapacitor containing a polyacrylamide hydrogel electrolyte, Angew. Chem., Int. Ed., 2017, 56(31), 9141-9145.

18 L. Han, K. Liu, M. Wang, K. Wang, L. Fang, H. Chen, J. Zhou and X. Lu, Mussel-inspired adhesive and conduc- 
tive hydrogel with long-lasting moisture and extreme temperature tolerance, Adv. Funct. Mater., 2018, 28(3), 1704195.

19 Q. Rong, W. Lei, L. Chen, Y. Yin, J. Zhou and M. Liu, Antifreezing, conductive self-healing organohydrogels with stable strain-sensitivity at subzero temperatures, Angew. Chem., Int. Ed., 2017, 56(45), 14159-14163.

20 F. Chen, D. Zhou, J. Wang, T. Li, X. Zhou, T. Gan, S. Handschuh-Wang and X. Zhou, Rational fabrication of anti-freezing, non-drying tough organohydrogels by onepot solvent displacement, Angew. Chem., Int. Ed., 2018, 57(22), 6568-6571.

21 X.-F. Zhang, X. Ma, T. Hou, K. Guo, J. Yin, Z. Wang, L. Shu, M. He and J. Yao, Inorganic salt induced thermal reversible and anti-freezing cellulose hydrogels, Angew. Chem., 2019, 58(22), 7366-7370.

22 T. Fujii, Y. Hata, M. Oozeki, H. Moriyama, T. Wakagi, N. Tanaka and T. Oshima, The crystal structure of zinc-containing ferredoxin from the thermoacidophilic archaeon Sulfolobus sp. strain 7, Biochemistry, 1997, 36(6), 15051513.

23 C. A. Smith, H. S. Toogood, H. M. Baker, R. M. Daniel and E. N. Baker, Calcium-mediated thermostability in the subtilisin superfamily: The crystal structure of Bacillus Ak.1 protease at 1.8 angstrom resolution, J. Mol. Biol., 1999, 294(4), 1027-1040.

24 C. Vieille and G. J. Zeikus, Hyperthermophilic enzymes: sources, uses, and molecular mechanisms for thermostability, Microbiol. Mol. Biol. Rev., 2001, 65(1), 1-43.

25 C. M. T. V. Tournier, A. Gilles, B. David, C. Folgoas, E. Moya-Leclair, E. kamionka, M.-L. Desrousseaus, H. Texier, S. Gavalda, M. Cot, E. Guemard, M. Dalibey, J. Nomme, G. Cioci, S. Barbe, M. Chateau, I. andre, S. Duquesne and A. Marty, An engineered PET depolymerase to break down and recycle plastic bottles, Nature, 2020, 580, 216-219.

26 Y. Qiao, Y. Lin, Z. Yang, H. Chen, S. Zhang, Y. Yan and J. Huang, Unique temperature-dependent supramolecular self-assembly: from hierarchical 1D nanostructures to super hydrogel, J. Phys. Chem. B, 2010, 114(36), 11725-11730.
27 J. Brassinne, J.-P. Bourgeois, C.-A. Fustin and J.-F. Gohy, Thermo-responsive properties of metallo-supramolecular block copolymer micellar hydrogels, Soft Matter, 2014, 10(17), 3086-3092.

28 S. Basak, I. Singh, A. Banerjee and H.-B. Kraatz, Amino acid-based amphiphilic hydrogels: metal ion induced tuning of mechanical and thermal stability, RSC Adv., 2017, 7(24), 14461-14465.

29 S. H. Zheng, Z. W. Li, C. X. Zhang, Y. Q. Li, L. Lin, Z. B. Yan, X. H. Zhou, Y. P. Wang, X. S. Gao and J. M. Liu, Two-level hierarchical stripe domains and enhanced piezoelectricity of rapid hot-press sintered BiFeO3 ceramics, J. Appl. Phys., 2018, 124(19), 194104.

30 Y.-M. Chung, K. L. Simmons, A. Gutowska and B. Jeong, Sol-gel transition temperature of PLGA-g-PEG aqueous solutions, Biomacromolecules, 2002, 3(3), 511-516.

31 Y. Qiao, Y. Y. Lin, S. Liu, S. F. Zhang, H. F. Chen, Y. J. Wang, Y. Yan, X. F. Guo and J. B. Huang, Metal-driven hierarchical self-assembled zigzag nanoarchitectures with electrical conductivity, Chem. Commun., 2013, 49(7), 704706.

32 Z. Wu, R. Xue, M. Xie, X. Wang, Z. Liu, M. Drechsler, J. Huang and Y. Yan, Self-assembly-triggered cis-to-trans conversion of azobenzene compounds, J. Phys. Chem. Lett., 2017, 9(1), 163-169.

33 X. Zhang, X. Guo, S. Yang, S. Tan, X. Li, H. Dai, X. Yu, X. Zhang, N. Weng and B. Jian, Double-network hydrogel with high mechanical strength prepared from two biocompatible polymers, J. Appl. Polym. Sci., 2009, 112(5), 30633070.

34 J. P. Gong, T. Kurokawa, T. Narita, G. Kagata, Y. Osada, G. Nishimura and M. Kinjo, Synthesis of hydrogels with extremely low surface friction, J. Am. Chem. Soc., 2001, 123(23), 5582-5583.

35 J. P. Gong, Why are double network hydrogels so tough?, Soft Matter, 2010, 6(12), 2583-2590.

36 J. P. Gong, Y. Katsuyama, T. Kurokawa and Y. Osada, Double-network hydrogels with extremely high mechanical strength, Adv. Mater., 2003, 15(14), 1155-1158. 